\title{
An Expert System for Plasma Cutting Process Quality Prediction and Optimal Parameter Suggestion
}

\author{
Sam Y. S. Yang \\ CSIRO Manufacturing Science and Technology, Australia \\ Em: s.yang@cmst.csiro.au
}

Keywords Plasma cutting, CNC, Information Infrastructure

\begin{abstract}
An hybrid expert system, which uses both rule-based reasoning and statistical modelling, is developed for prediction of cut quality and suggestion of optimal process parameter settings for CNC controlled plasma metal-plate cutting machines. A scheme for process parameter classification has been developed. They are classified according to whether they are fixed, operator selectable or adjustable. A scheme for cutting quality definition and practical method of assessment have been developed which incorporate both the quantitative requirements for each quality attribute and their respective relative importance. When process parameter values are entered, the system gives the expected quality outcome. When the quality requirements are specified, the system outputs the optimal parameter setting values. The prototype of the system has been tested and the predictions are consistent with experimental measurements.
\end{abstract}

\section{INTRODUCTION}

Plate cutting is one of the most important manufacturing processes for metal components making. The quality and the cost of cutting process is often critical for final product quality and cost. It is estimated that the plasma cutting machine with a computer numerical controlled (CNC) torch movement is the optimal choice for $80 \%$ of the metal plate cutting processes [1]. However, the quality of the plasma cutting process is often unpredictable largely due to the unknown effects of the different process parameters. Difficulty in quality control is one of the factors which affect the wide spread use of the plasma cutting process in industry. 
Requirements for quality control and prediction are quite common in other manufacturing industries as well. Various expert system approaches have been used to tackle them [2-3]. The hybrid expert system [4], which combines both the shallow-knowledge (rule based) approach with the deepknowledge (modelling and simulation) approach, has been used in various industrial processes [5].

This paper outlines the design and formulation of an expert system for the metal plate plasma cutting process. The system predicts cutting quality for a given process parameter setting, and provides advice on the optimal process parameter setting for a given quality requirement. The major tasks include definition and classification of process parameters, definition and representation of cut quality, knowledge capturing, data analysis and modelling, representation and delivering of knowledge in a form understandable by the users.

The software modules described in this paper form parts of a larger system for Remote Operations, Diagnostics And Maintenance (ROSDAM). The modules to be discussed include quality prediction and optimal parameter values suggestion modules under operation support, and process knowledge database maintenance module under database maintenance.

\section{PROCESS VARIABLES AND CUT QUALITY}

The first task is to find manageable methods and definitions for describing the input and output parameters of the system. This involves the definition of a complete set of process parameters and the cut quality in a quantifiable and repeatable way.

The process parameters are classified into three categories: fixed, selectable and adjustable. A fixed parameter is the one which cannot be modified for a given cutting job, such as the model of the cutting equipment. A selectable parameter is one that the operator can select from a discrete range of options, such as the type of plasma gas. Adjustable parameters are those parameters which can be set in a range of numerical values, such as the contouring speed of the cutting torch. For the plasma cutting process, there are a total of 120 process parameters.

The process parameters can also be classified according to whether they have a natural numerical representation or not. The parameters which have such a representation include plate thickness, cutting current, consumable condition (a lower value represent a better condition), etc.. The nonnumerical parameters, such as machine model, plate material and grade, are used to sub-classify the process. Mathematically, the process parameters are represented as a parameter vector 


$$
P^{(\alpha)}=\left\{p_{1}^{(\alpha)}, p_{2}^{(\alpha)}, \cdots, p_{N}^{(\alpha)}\right\}
$$

where the subscript denotes the numerical parameters with $N$ being the total number of such parameters, and $\alpha$ denotes the non-numerical parameters.

Cut quality not only includes measurable qualities such as cut angle and accuracy, but also has abstract entities such as dross removability and surface finish. As the system is intended for actual use in industrial environment, the measurement procedures to obtain some level of accuracy in these qualities has been considered.

Even if the individual quality attribute can be quantified, it is often the case that there is not a universal definition for "good" quality. Whether a particular cut is good quality or not relates to the part to be made. For instance, if the part is for a drop-in fit, the cut size cannot be larger than the design value. If the part needs be joined to other parts in a given angle, the "cut angle" is critical. If the plate need to be joined by welding, the "nitrition" is a important factor. If the cutting surface forms part of the product finish, the surface finish may be the main concern [6]. To describe this job-dependent quality definition, a weighting factor is introduced in our system.

For plasma cutting, a total of eight quality attributes are considered. They include part size accuracy, cut angle, cut surface finish, dross peripheral coverage and removability. They are defined by their averages and standard deviations, and a relative weighting factor for each quality attribute which are represented by the following three vectors

$$
\left\{\begin{array}{l}
Q=\left\{q_{1}, q_{2}, \cdots, q_{M}\right\} \\
\delta Q=\left\{\delta q_{1}, \delta q_{2}, \cdots, \delta q_{M}\right\} \\
W=\left\{w_{1}, w_{2}, \cdots, w_{M}\right\}
\end{array}\right.
$$

where $q_{i}, \delta q_{i}$ and $w_{i}(i=1,2, \ldots, M)$ are the average, standard deviation and weighting factor for the $i$ 'th quality attribute. $M(=8)$ is the total number of such quality attributes. A "good" cut is the one with averages of quality attributes close to the expected values and the variations (proportional to the respective standard deviations) are within specified limits.

The expert system to be described in this paper is based on the above definitions for process parameters and quality descriptions. The expert system has a user-friendly GUI for plasma cutting process data collection. When a cut job is completed, the machine operator can easily input the process parameters and the associated quality into the system. In the following sections, we will focus on the principles of quality prediction and optimal parameter suggestions. 


\section{CUT QUALITY PREDICTION}

One of the functions of the expert system is to predict cut quality when the process parameter values are specified by the user. The architecture of the system is represented schematically as in Figure 1.

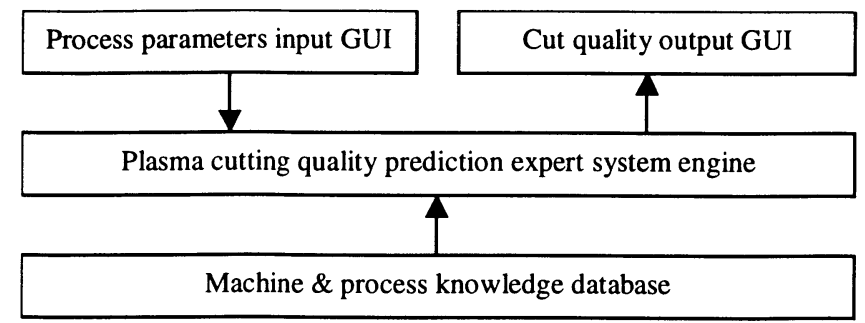

Figure 1: Block diagram of the expert system module for quality prediction.

Process parameter values are entered in to the system by the user through the process parameter input GUI. The plasma cutting quality prediction expert system engine uses the user input to search for the appropriate information in the machine and process knowledge database. The information enables the system to work out the expected quality. The predicted quality values are sent to the cut quality output GUI.

The quality prediction is based on the assumption that the change to quality vector $\Delta Q$ is small for a small change to the process parameter vector $\Delta P$. Consequently, they can be related through a Jacobian or slope matrix $S$ as in the following:

$$
\Delta Q=S \Delta P
$$

The matrix $S$ in (3.1) is part of the process knowledge which is stored in the process knowledge database. The values of the matrix elements are obtained through a database maintenance process. The maintenance process can be represented by the block diagram as shown in Figure 2 .

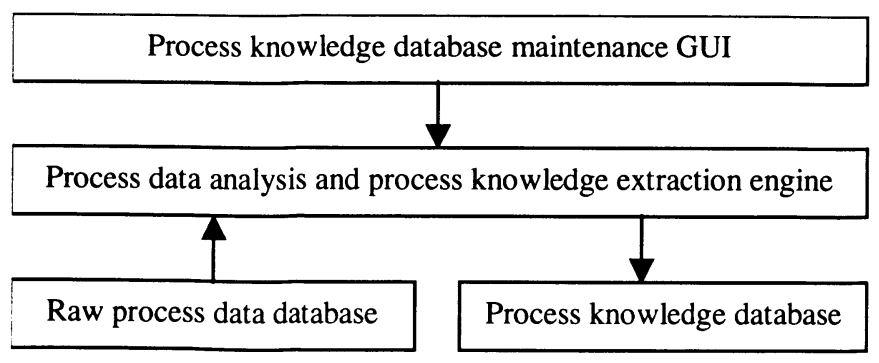

Figure 2: Block diagram for process knowledge database maintenance. 
The process knowledge database maintenance process is initiated by the user when a batch of new raw process data has been accumulated in the raw process data database. The process data analysis and process knowledge extraction engine calculates the slope matrix for each entry of raw process data, and output the relevant process information and the slope matrix $S$ to the process knowledge database.

The system has been populated and trained with 150 process data sets for a Hypertherm HT2000 plasma machine controlled by a FARLEY PDF32/Wizard II CNC. Another seven sample coupons have been cut and their quality attribute values were measured experimentally. All coupons were cut on $6 \mathrm{~mm}$ grade 250 mild steel BHP plates. The cutting gas is oxygen and the shield gas is air. Dry cutting bed is used and no magnetic handling is used for the plates. New Hypertherm standard 200A consumables are used for the coupon cuts.

For the above cutting conditions, the HyperTherm manual recommended a cutting speed of $4.06 \mathrm{~m} / \mathrm{min}$. The other cutting parameter values as recommended by HyperTherm are shown in the first row of Table 1. Due to software system limitations, the speed setting only preserves the first decimal place. In Table 1, each row corresponds to a test coupon. The parameters in Table 1 were so selected as they are commonly regarded in industry as the main influential plasma cutting parameters. It should be noted that these test coupons are used merely to verify the system predictions. They should not be mixed with the well-known design of experiments methodology for determining how quality depends on process parameter variations.

Table 1: Cut process parameter set values for test samples.

\begin{tabular}{ccccccc}
\hline $\begin{array}{c}\text { Sample } \\
\text { number }\end{array}$ & $\begin{array}{c}\text { Current } \\
(\mathbf{A})\end{array}$ & $\begin{array}{c}\text { Voltage } \\
(\mathbf{V})\end{array}$ & $\begin{array}{c}\text { Speed } \\
(\mathbf{m} / \mathbf{m i n})\end{array}$ & $\begin{array}{c}\text { Cut flow } \\
(\mathbf{\%})\end{array}$ & $\begin{array}{c}\text { Shield } \\
\text { pressure } \\
(\mathbf{p s i})\end{array}$ & $\begin{array}{c}\text { Kerf } \\
\text { comp } \\
(\mathbf{m m})\end{array}$ \\
\hline 1 & 200 & 120 & 4.1 & 64 & 60 & 1.25 \\
\hline 2 & 180 & 120 & 4.1 & 64 & 60 & 1.25 \\
\hline 3 & 200 & 130 & 4.1 & 64 & 60 & 1.25 \\
\hline 4 & 200 & 120 & 4.4 & 64 & 60 & 1.25 \\
\hline 5 & 200 & 120 & 4.1 & 51 & 60 & 1.25 \\
\hline 6 & 200 & 120 & 4.1 & 64 & 40 & 1.25 \\
\hline 7 & 200 & 120 & 4.1 & 64 & 60 & 0.00 \\
\hline
\end{tabular}

The experimentally measured quality values and the values predicted by the system are listed in Tables 2 . It is seen in the table that the predictions agree reasonably well with experimental measurements. The precision of the prediction will improve as the system is trained with more data sets. 


\section{PROCESS PARAMETER OPTIMIZATION}

Ultimately, what the user want to know is about how to set up the machine parameters for a given quality requirement. The process parameter optimisation module of the system is developed to meet this user requirement. The module has an input GUI for the user to specify the cutting environment such as machine model, plate specifications, range of gas type selections, etc, and the quality expectation. The quality expectation by the user for each quality attribute is defined by its expected (average) value, a range which must be satisfied and a weight factor as defined in (2.2). The logical structure of the module is shown schematically in Figure 3.

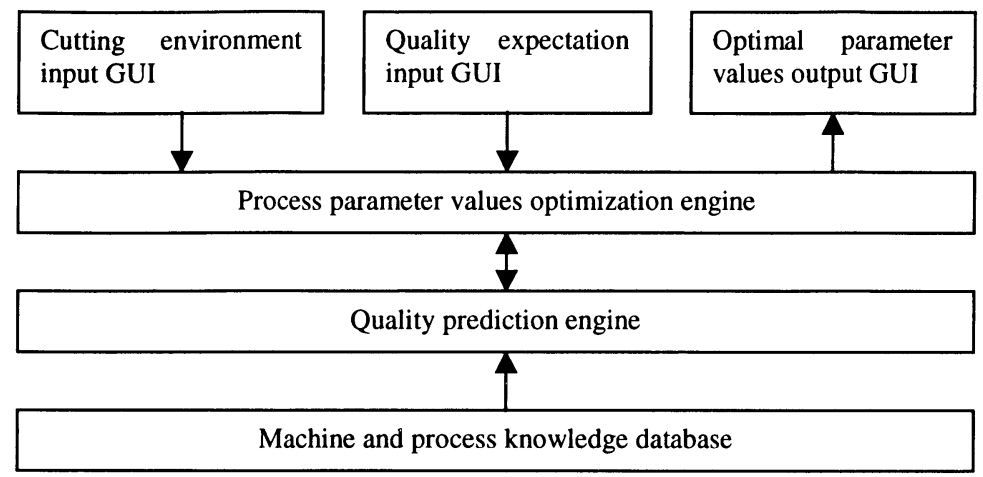

Figure 3: Block diagram for optimal parameter values suggestion

The process parameter values optimisation engine calls the quality prediction engine to calculate the expected quality output. By adjusting the parameter values, the process optimisation engine minimises the target function as defined in the following:

$$
T=\frac{1}{M} \sum_{i=1}^{M} w_{i}\left|q_{i}-\hat{q}_{i}\right| / \delta_{i}
$$

where $w_{i}$ is the user supplied weight factor for the $i$ 'th quality attribute, $q_{i}$ and $\hat{q}_{i}$ are the predicted and the user specified values for the $i$ 'th quality attribute, and $\delta_{i}$ is the typical amplitude of variation for the $i$ 'th quality attribute.

The optimisation is subject to the following constraints:

$$
\left\{\begin{array}{l}
q_{i}^{(\min )} \leq q_{i}-\Delta q_{i} \\
q_{i}^{(\max )} \geq q_{i}+\Delta q_{i}
\end{array} \quad i=1,2, \cdots, M\right.
$$


Table 2

Comparison of predicted and experimentally measured quality attribute values for the test samples. The lower bound (LB) and upper bound $(U B)$ are average minus/plus standard deviations for predicted values and minimum and maximum for experimentally measured values.

\begin{tabular}{|c|c|c|c|c|c|c|c|}
\hline \multirow{2}{*}{$\begin{array}{l}\text { Quality } \\
\text { attribute }\end{array}$} & \multirow{2}{*}{$\begin{array}{c}\text { Sample } \\
\text { numbe } \\
\mathbf{r}\end{array}$} & \multicolumn{3}{|c|}{ Predicted quality values } & \multicolumn{3}{|c|}{ Measured quality values } \\
\hline & & Averag & LB & UB & Averag & LB & UB \\
\hline \multirow{5}{*}{$\begin{array}{c}\text { Top size } \\
\text { error }(\mathrm{mm})\end{array}$} & 1 & -1.42 & -1.81 & -1.03 & -1.40 & -1.48 & -1.32 \\
\hline & 2 & -1.36 & -1.68 & -1.04 & -1.36 & -1.53 & -1.19 \\
\hline & 3 & -0.41 & -4.22 & 3.40 & -1.74 & -1.45 & -2.01 \\
\hline & 4 & -1.40 & -1.79 & -1.01 & -1.51 & -2.35 & -1.07 \\
\hline & 5 & -1.51 & -2.02 & -1.00 & -1.53 & -2.11 & -1.27 \\
\hline \multirow{5}{*}{$\begin{array}{c}\text { Bottom size } \\
\text { error } \\
(\mathrm{mm})\end{array}$} & 1 & 0.24 & 0.01 & 0.46 & 0.39 & 0.20 & 0.58 \\
\hline & 2 & 0.54 & 0.19 & 0.90 & 0.51 & 0.15 & 0.87 \\
\hline & 3 & -1.85 & -8.15 & 4.45 & 0.17 & -0.06 & 0.20 \\
\hline & 4 & 0.33 & 0.01 & 0.66 & 0.41 & 0.16 & 0.65 \\
\hline & 5 & 0.31 & -0.38 & 1.00 & 0.34 & 0.27 & 0.54 \\
\hline \multirow{5}{*}{$\begin{array}{l}\text { Cut angle } \\
\text { (degree) }\end{array}$} & 1 & 6.27 & 4.89 & 7.65 & 6.40 & 4.63 & 8.17 \\
\hline & 2 & 7.10 & 5.96 & 8.24 & 7.10 & 4.58 & 9.62 \\
\hline & 3 & 1.20 & -17.29 & 19.69 & 7.37 & 0.47 & 6.73 \\
\hline & 4 & 6.41 & 5.03 & 7.79 & 6.67 & 5.37 & 8.31 \\
\hline & 5 & 7.04 & 3.92 & 10.15 & 6.30 & 5.60 & 7.41 \\
\hline \multirow{5}{*}{$\begin{array}{l}\text { Surface } \\
\text { finish } \\
\text { (grade) }\end{array}$} & 1 & 2 & 2 & 3 & 2 & - & - \\
\hline & 2 & 2 & 2 & 3 & 3 & - & - \\
\hline & 3 & 1 & 1 & 5 & 3 & - & - \\
\hline & 4 & 2 & 1 & 3 & 3 & - & - \\
\hline & 5 & 3 & 1 & 5 & 3 & - & - \\
\hline \multirow{5}{*}{$\begin{array}{c}\text { Dross } \\
\text { removability } \\
\text { (grade) }\end{array}$} & 1 & 3 & 3 & 3 & 3 & - & - \\
\hline & 2 & 3 & 3 & 3 & 3 & - & - \\
\hline & 3 & 2 & 1 & 3 & 3 & - & - \\
\hline & 4 & 3 & 2 & 4 & 3 & - & - \\
\hline & 5 & 3 & 3 & 4 & 3 & - & - \\
\hline \multirow{5}{*}{$\begin{array}{c}\text { Dross } \\
\text { peripheral } \\
\text { coverage } \\
(\%)\end{array}$} & 1 & 0 & 0 & 8 & 0 & - & - \\
\hline & 2 & 9 & 0 & 30 & 0 & - & - \\
\hline & 3 & 0 & 0 & 100 & 0 & - & - \\
\hline & 4 & 0 & 0 & 9 & 0 & - & - \\
\hline & 5 & 6 & 0 & 42 & 0 & - & - \\
\hline \multirow{5}{*}{$\begin{array}{l}\text { Top kerf } \\
\text { width } \\
\text { (mm) }\end{array}$} & 1 & 3.37 & 3.28 & 3.47 & 3.35 & 3.21 & 3.49 \\
\hline & 2 & 3.32 & 3.22 & 3.42 & 3.37 & 3.27 & 3.47 \\
\hline & 3 & 4.17 & 2.24 & 6.10 & 3.63 & 3.58 & 3.78 \\
\hline & 4 & 3.34 & 3.13 & 3.55 & 3.42 & 3.35 & 3.54 \\
\hline & 5 & 3.35 & 3.02 & 3.69 & 3.41 & 3.33 & 3.47 \\
\hline \multirow{5}{*}{$\begin{array}{l}\text { Bottom kerf } \\
\text { width } \\
(\mathrm{mm})\end{array}$} & 1 & 1.66 & 1.56 & 1.76 & 1.81 & 1.75 & 1.87 \\
\hline & 2 & 1.54 & 1.47 & 1.60 & 1.61 & 1.56 & 1.66 \\
\hline & 3 & 1.56 & 0.04 & 3.07 & 1.70 & 1.68 & 1.76 \\
\hline & 4 & 1.53 & 1.36 & 1.71 & 1.63 & 1.61 & 1.64 \\
\hline & 5 & 1.54 & 1.18 & 1.90 & 1.63 & 1.58 & 1.67 \\
\hline
\end{tabular}

where $q_{i}^{(\min )}$ and $q_{i}^{(\max )}$ are user specified range and $\Delta q_{i}$ is the predicted variation for the $i$ 'th quality attribute. The optimal cutting parameters are 
obtained through the above optimisation process. The output from the process optimisation module is the optimal choice of the process parameters for the given quality requirement.

As can be seen in the above discussions, the accuracy and reliability of the process parameter suggestion engine depends heavily on the performance of the quality prediction engine. Test with the existing small process data set produced output which agrees reasonably with opinions of the experienced machine operators.

\section{CONCLUSION}

This paper described the design of a hybrid expert system for plasma metal plate cutting machines. The system incorporates both the rule-based reasoning and the modelling/simulation. The quality predictions parameter suggestion from the prototype agree well with experimental measurements, even though the system is only been trained with a small number of process data sets at this stage. It is expected that the accuracy of the prediction will improve after the system has been trained by a larger number of process data sets. Use of the system is expected to reduce quality uncertainties and machine set up time. This will leads to improvements in cut quality and reduction in production cost.

The construction of the system and the methods involved are quite general and should be applicable to other industrial processes with appropriate modifications.

\section{REFERENCES}

[1] FABCUT - The Fabrication and Cutting Bulletin (July 1996) 1 "One in five" may regret buying laser

[2] J. P. T. Mo, C. Menzel, Computers in Industry 37 (1998) 171-183 An integrated process model driven knowledge based system for remote customer support

[3] W. B. Rowe, Y. Li, B. Mills, D. R. Allanson, Computers in Industry 31 (1996) 45-60 Application of intelligent CNC in grinding

[4] M. Kleiber, Z. Kulpa, CAMES 2 (1995) 165-186 Computer-assisted hybrid reasoning in simulation and analysis of physical systems

[5] Z. Kulpa, A. Radomski, O. Gajl, M. Kleiber, I. Skalna, Engineering Applications of Artificial Intelligence 12 (1999) 229-240 Hybrid expert system for qualitative and quantitative analysis of truss structures

[6] Welding Design and Fabrication (Dec 1998) 7-27 Cutting equipment 